\title{
The Impact of Laparoscopic Treated Endometrioma on the Live Birth Rate in IVF/ICSI Cycles Compared with Unexplained Infertility: A Prospective Randomized Study
}

\author{
Snezhana Stojkovska ${ }^{1 *}$ D, Gligor Dimitrov ${ }^{1}$, Jane Stojkovski $^{1}$, Stefan Saltirovski $^{2}$, Makuli Hadzi Lega $^{1}$ \\ ${ }^{1}$ Department of IVF and Reproductive Medicine, Remedika, Skopje, Republic of Macedonia; ${ }^{2 K i n d e r w u n s c h, ~ K i e l, ~ G e r m a n y ~}$
}

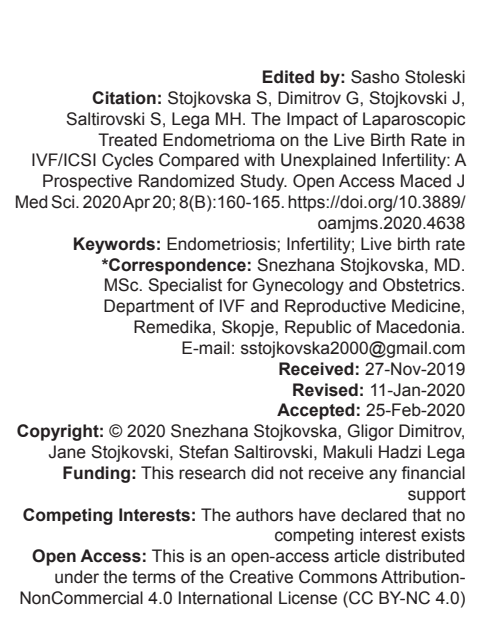

Abstract

BACKGROUND: It is estimated that $30-70 \%$ of patients who undergo treatment for infertility are afflicted with endometriosis.

AIM: The objectives of this study are to evaluate the impact of laparoscopic treated endometrioma compared to unexplained subfertility on the live birth rate in women undergoing in vitro fertilization (IVF)/intracytoplasmic sperm injection (ICSI).

METHODS: This randomized prospective study included 120 women who contacted the department of IVF in the period from 2010 to 2015 . Women were divided into two groups according to the findings obtained by laparoscopy. The treated endometrioma group $(n=60)$ with unilateral ovarian endometriomas and the non-endometriosis group $(n=60)$ with unexplained infertility undergoing the first cycle of IVF-embryo transfer (IVF-ET) were included in the study. In all participants, ICSI was used and all had fresh embryo transfer per cycle. The primary outcome was to live birth.

RESULTS: Our results demonstrated that clinical pregnancy rates $(p=0.54)$ and live birth rate $(p=0.63)$ are similar The preservation of a good ovarian response to stimulation by gonadotropins after laparoscopic ovarian cystectomy was presented. Laparoscopic cystectomy is followed by good IVF/ICSI outcome into the level expected in women with unexplained subfertility.

CONCLUSION: Therefore, operative treatment is justified by not altering the live birth rate. Additional study is needed to be considered cystectomy before IVF as an effective approach for managing endometriosis-associated infertility.

\section{Introduction}

Endometriosis is a complex disorder that affects $5-10 \%$ of the female population of reproductive age. The exact incidence or prevalence is difficult to determine. Higher incidences of endometriosis have been reported in patients who undergo treatment for infertility and approximately $30-70 \%$ of patients with endometriosis may suffer from infertility [1]. About $60-75 \%$ of cases are ovarian endometriomas. Ovarian endometriomas $>3 \mathrm{~cm}$ require adequate surgical treatment [2]. Surgery carries a risk of removing part of the normal ovarian tissue. The literature describes two techniques: Laparoscopic ovarian cystectomy, which involves stripping the cyst wall and laparoscopic vaporization of the internal layers of the endometriomas wall. The impact of endometriosis on fertility is not yet clear. Many factors have been suggested to cause infertility in women with endometriosis, including mechanical, immunological, and endocrinological [3], [4].

Using in vitro fertilization (IVF) treatment in patients with endometriosis can bypass certain disorders of the reproductive function. A number of studies report the role of ovarian endometriosis and its influence in the outcome of IVF fertilization. Data on the impact of endometriosis on the results of IVF treatment are controversial. Poor response to ovarian stimulation decreased oocyte retrieval rates, decreased fertilization, and implantation rates within the parameters that monitor the success of the IVF procedure have all been implicated. Several studies have reported that laparoscopic surgery of ovarian endometriosis can damage the ovarian reserve implicated a possibility of lower ovarian response to gonadotropins [5].

One Cochrane review compared the reproductive outcomes between laparoscopic surgical interventions and diagnostic laparoscopy in patients with minimal to mild endometriosis. The metaanalysis demonstrated an advantage of laparoscopic surgery compared to diagnostic laparoscopy in clinical pregnancy rate and live birth rate [6]. Furthermore, randomized control trials of surgical or expectant management for endometriosis prior IVF assessed the effectiveness of surgery for improving reproductive outcome [7]. In addition, the European Society of Human Reproduction and Embryology (ESHRE) guideline suggested that laparoscopic ovarian cystectomy prior IVF is recommended if an ovarian endometrioma is $4 \mathrm{~cm}$ or more in diameter with improve access to follicles and possibly improves the ovarian response [8]. 
However, results retrieved from studies and meta-analysis depicts a diverse pattern of IVF success, underlying the involvement of individual parameters in the appearance of the final outcome [9]. There is an insufficient data in the literature, which compared IVF outcome in women with treated endometriosis and women with other diagnostic entities for infertility. The aim of our study was to investigate whether laparoscopic treated endometrioma may adversely affect on the results of IVF/intracytoplasmic sperm injection (ICSI) outcomes with particular reference on the clinical pregnancy rate per embryo transfer cycle and live birth rate.

\section{Materials and Methods}

\section{Patient selection}

The randomized prospective study encompassed the period from 2010 to 2015, during which 120 patients were performed. Exclusion criteria for the women were endometriosis Stage IV; serum concentration of follicle-stimulating hormone (FSH) $>15 \mathrm{IU} / \mathrm{l}$; older than 41 year; with an abnormal ovarian reserve (antral follicle count $<5-7$ follicles or antiMullerian hormone $<0.5-1.1 \mathrm{ng} / \mathrm{mL}$ ), with a previous poor ovarian response; women with additional causes for infertility such as polycystic ovarian syndrome, uterine malformation, underlying immune conditions, as well as patients with infectious disease such as human immunodeficiency virus. Inclusion criteria for the women were minimum 2 years of infertility, regular menstrual cycle (intervals 25-35), normal serum concentration or prolactin, free thyroxin, and thyroid-stimulating hormone. The patients who had undergone laparoscopic surgery for endometriosis and who failed to become pregnant within 1 year after surgery have been evaluated. Only patients with laparoscopic excision of the ovarian endometrioma cyst were included in this study group. The non-endometriosis group was patients with unexplained subfertility who underwent IVF/ICSI during the same period of time. All patients had a diagnostic or surgical laparoscopy. Only women whose partners' have normal semen analyses were reviewed. Only cycles with long luteal protocol for IVF and ICSI were included in the study. In all participants, ICSI was used and all had fresh embryo transfer per cycle.

\section{Study group}

Endometriosis was diagnosed by laparoscopy and scored according to the revised classification of the American Fertility Society [10]. Patients were divided into two groups according to the findings obtained by laparoscopy. The endometriosis group of patients (60) was with ovarian endometriomas $>3 \mathrm{~cm}$, treated by cystectomy with stripping technique.
The non-endometriosis group $(n=60)$ was with unexplained subfertility. The laparoscopic findings in the group were normal, with no pathology of gynecologic organs. We have analyzed the parameters for ovarian stimulation with gonadotropins in a long luteal protocol and parameters that define the results of IVF fertilization. Such data would be useful for patient counseling and will supply further information for the impact of endometriosis on pregnancy rate in contemporary IVF/ICSI treatment.

\section{Ovarian stimulation}

A long stimulation protocol was used in all patients. Downregulation was initiated with buserelin acetate (Suprefact, Sanofi Aventis, Kallithea, Athens, Greece) during the mid-luteal phase. Injections of recombinant follitropin beta (Puregon, N.V. Oregon, OS, The Netherlands) were initiated on the $3^{\text {rd }}$ day of the menstrual cycle. The doses were adjusted to the patient's age and the numbers and size of follicles and the estradiol level. We followed during the controlled ovarian stimulation a protocol set by our IVF department. With ultrasonography and laboratory tests, we evaluated several parameters such as estradiol $\left(E_{2}\right)$, number and size of follicles, endometrium thickness, and gonadotropins administration. We started with $\mathrm{rFSH}$ administration on the $3^{\text {rd }}$ day of the menstrual cycle when the $E_{2}$ level was $<30 \mathrm{pg} / \mathrm{ml}$. When the estradiol level was $\pm 200 \mathrm{pg} / \mathrm{ml}$ per follicle $\geq 18 \mathrm{~mm}$ in diameter, chorionic gonadotropin (human chorionic gonadotropin injection [hCG], 10,000 IU; Pregnyl, N.V. Organon, OS, The Netherlands) was administered for triggering the maturation of oocytes.

\section{Ethical consideration}

This randomized prospective study was approved by the Clinical Research and Research Ethics Committees (code: 455). We obtain from all participants written consent.

\section{Statistical analysis}

Statistical analysis of the data was performed in the statistical program Statistica 7.1. The analysis of the series with attributive marks, percentages of the structure (\%), was determined and tested using the Pearson's Chi-square test and the exact Fisher test (p). Descriptive statistics were used for the distribution of data (Kolmogorov-Smirnov test; Lilliefors test; and Shapiro-Wilks test). The age difference between patients was tested with analysis of variance $(F ; p) /$ Tukey post hoc $(p)$. The difference in the analyzed parameters between patient groups in case of deviation from a normal distribution was tested with Kruskal-Wallis ANOVA by ranks $(H)$. The statistical difference in the analyzed parameters between two groups of patients 
was tested with Mann-Whitney U-test (Z). Fertilization rate, implantation rate, clinical pregnancy rate, and the birth rate in all groups (\%) were made. Differences were considered significant when $p<0.05$.

\section{Results}

Patient characteristics are shown in Table 1. There were no significant differences in age $F=1.54$ and $p>0.05(p=0.34)$, duration of infertility $F=1.97$ and $p>0.05(p=0.27)$, or body mass index $F=1.63$ and $p>0.05(p=0.19)$ among the groups.

Table 1: Baseline characteristics of patients $(n=120)$

\begin{tabular}{lllc}
\hline Characteristics & $\begin{array}{l}\text { Endometrioma } \\
\text { cystectomy (60) }\end{array}$ & $\begin{array}{l}\text { Unexplained } \\
\text { subfertility (60) }\end{array}$ & $\mathrm{p}$-value \\
\hline Age (years) & $32.10 \pm 4.35$ & $31.20 \pm 3.81$ & 0.34 \\
Body mass index & $24.2 \pm 3.5$ & $21.8 \pm 2.6$ & 0.19 \\
Duration of infertility (years) & $4.7 \pm 2.3$ & $4.0 \pm 2.6$ & 0.27 \\
\hline Chi-square test for categorical and Student's t-test for continuous data. $\mathrm{p}>0.05$ compared to unexplained
\end{tabular}

The mean age was not more than 36 years, an important role in the further processing of data. The female age is undoubtedly the most significant factor influencing the IVF outcome. In addition, in all patients included in the study, a serum anti-Mullerian hormone was in reference value before surgery. In the parameters for ovarian stimulation, no significant statistical differences appear in all four groups. Descriptive statistics of the level of estradiol, the day of hCG administration, the number of ampoules, and the thickness of the endometrium were very similar. In this study, we remarked that in endometriosis patients with ovarian cystectomy, a longer stimulation with gonadotropin was required, and at the same time, more ampoules were used, but without any statistical significance $p>0.05(p=0.35)$. Using descriptive statistics, it was noted that the average number of mature oocytes (MII) was lower in patients with ovarian cystectomy $(7.70 \pm 2.91)$, compared to unexplained subfertility group, but both parameters were without any statistical significance $p>0.05(p=0.13)$ (Table 2).

Table 2: Ovarian stimulation parameters $(n=120)$

\begin{tabular}{llll}
\hline Characteristics & $\begin{array}{l}\text { Endometrioma } \\
\text { cystectomy }(60)\end{array}$ & $\begin{array}{l}\text { Unexplained } \\
\text { subfertility }(60)\end{array}$ & p-values \\
\hline Total gonadotropin dose (units) & $2711.40 \pm 16.91$ & $2250.50 \pm 14.38$ & 0.35 \\
Follicles & $9.17 \pm 4.62$ & $14.33 \pm 7.50$ & 0.21 \\
Mature oocytes & $7.70 \pm 2.91$ & $11.37 \pm 4.13$ & 0.13 \\
Estradiol peak & $1515.53 \pm 517.10$ & $1598.33 \pm 521.11$ & 0.65 \\
$\begin{array}{l}\text { Duration of ovarian stimulation } \\
\text { (days) }\end{array}$ & $10.29 \pm 2.30$ & $9.63 \pm 2.61$ & 0.71 \\
\hline $\begin{array}{l}\text { Chi-square test for categorical and Student's t-test for continuous data. p>0.05 compared to unexplained } \\
\text { subertilyy. }\end{array}$
\end{tabular}

In terms of the parameters of IVF outcome, no significant statistical correlations were found between the groups. This study clearly showed that fertilization rates were not affected in patients undergoing laparoscopic excisional surgery for ovarian endometrioma. Clinical pregnancy rate between groups, respectively, varying from $53.3 \%$ to $35.90 \%$ and the live birth rate $43.3 \%$ to $50 \%$ (Table 3).
Table 3: In vitro fertilization outcome

\begin{tabular}{llll}
\hline Characteristics & $\begin{array}{l}\text { Endometrioma } \\
\text { cystectomy (60) }\end{array}$ & $\begin{array}{l}\text { Unexplained } \\
\text { subfertility (60) }\end{array}$ & p-values \\
\hline Fertilization rate (\%) & 77.06 & 70.38 & 0.67 \\
Embryos/cycle & $5.93 \pm 4.09$ & $8.01 \pm 7.96$ & 0.83 \\
Transferred embryos/cycle & $2.30 \pm 1.53$ & $2.60 \pm 1.13$ & 0.79 \\
Implantation rate per embryo transfer (\%) & 30.43 & 35.90 & 0.43 \\
Pregnancy rate per embryo transfer & 53.3 & 35.90 & 0.54 \\
$\begin{array}{l}\text { cycle (\%) } \\
\text { Live birth rate (\%) }\end{array}$ & 43.3 & 50 & 0.63 \\
\hline Data presented as breakdown and one-way ANOVA. p>0.05 compared to unexplained subfertility.
\end{tabular}

The results among groups were analyzed, but they remained without any statistical significance. None of the patients experienced any complications from controlled ovarian stimulation, trigger, or oocyte retrieval.

\section{Discussion}

The high prevalence of endometriosis imposes the need for correct management between infertile women. There is still no consensus on the impact of endometriosis on IVF outcome and whether ovarian endometrioma should be removed in subfertility women before IVF.

Harb et al. published a systematic review and meta-analysis on the effect of endometriosis on IVF outcome [11]. Studies compared IVF outcome in women with endometriosis and women without endometriosis. This meta-analysis showed that fertilization rates were reduced in the stage of minimal and mild endometriosis, and there was a decrease in the implantation rate and clinical pregnancy rate in women with the stage of moderate and severe endometriosis undergoing IVF treatment. The explanation is found in abnormal endometrial pinopode formation, an embryotoxic milieu, and progesterone resistance in women with endometriosis [12], [13], [14]. These factors play a crucial part in reducing fertility in women with endometriosis.

In Harb review, a $14 \%$ reduction in live birth rates was observed in patients with stage III/IV endometriosis. However, this did not reach statistical significance.

Furthermore, Hamdan et al. were noticed in his review significantly higher cancellation rate in women with endometriosis undergoing IVF/ICSI in contrast with those without endometriosis. [15]. Furthermore, baseline FSH levels were higher when compared with women with no endometriosis although AFC and total stimulation dose were comparable between bought groups. In terms of the impact on surgical intervention for endometrioma on IVF/ICSI outcome, many studies evaluated in Hamdan review demonstrated similar clinical and live births between surgically treated endometrioma versus women with intact endometrioma. Women with endometrioma who had surgical treatment had besides a lower AFC and required higher total gonadotropin stimulation dose. Both groups had similar baseline FSH. In addition, 
women with surgically treated endometrioma versus normal contralateral ovary had retrieved a lower number of oocytes from the treated ovary. There is no doubt that surgery on endometriosis has a detrimental impact on ovarian reserve [16]. Therefore, it is justified to evaluate ovarian reserve before operative treatment even in younger patients. Individualization of care for women with endometriosis before IVF/ICSI may help optimize their IVF/ICSI results [17].

There is a general consensus that IVF should be recommended in infertile women who fail to become pregnant after surgical treatment [9]. A number of studies have taken into consideration the impact of IVF in pregnancy rate in patients with surgically treated endometriosis. Namely, the study of Dechanet presented that the surgery before IVF increasing the pregnancy rate in women with endometriomas [18]. Gonadotropin-releasing hormone $(\mathrm{GnRH})$ agonists have also been reported to increase pregnancy rate compared to GnRH antagonists [19]. The recent study showed that surgery for big endometrioma could be required before IVF to facilitate access to follicles during oocyte retrieval or to improve the ovarian response to controlled ovarian stimulation. Prolonged pituitary downregulation in women with surgically treatment endometriosis may be helpful to increase the clinical pregnancy rate in subsequent IVF cycles [20]. This integrated approach (surgery and IVF-ET) produced a pregnancy rate of $56.1 \%$ compared to a significantly lower pregnancy rate of only $37.4 \%$ after surgery alone [21].

Crochet performed a retrospective casecontrol study, to investigate the progression of endometriosis after IVF treatment in women having previously undergone surgical intervention [22]. The findings suggested that IVF is not responsible for the aggravation of endometriosis. During the controlled ovarian stimulation, it was shown that the pregnancy rate, oocytes, and functionality of the embryo were not affected by the presence of endometriomas, regardless of whether the endometriomas were surgically treated or not [23]. The pregnancy rate is negatively associated with the degree of endometriosis and the optimal time for the IVF procedure [24]. It is considered to be between 7 and 25 months after surgery.

Regarding the risk of miscarriage when endometriosis is present, IVF appears to be an independent factor [25]. Care should also be taken to endometriosis fertility index (EFI) because it represents a new staging system and has proved to be a useful tool in the hands of doctors [26]. EFI is used to predict fertility sequentially to surgery. Regarding the women who underwent surgery, a follow-up assessment of EFI $\geq 5-12$ months postoperatively was indicated the implementation of IVF-ET procedure [27].

Our study has highlighted the lack of clinical studies examining the surgically treated endometrioma with women with no endometriosis. The study was in line with ESHRE guidelines on endometrioma management. With surgery, women not only get relief from clinical symptoms but also there is better accessibility of the ovaries for oocyte retrieval. Furthermore, better results after surgically treated endometriosis can be expected as a result of the restoration of normal anatomy. We noticed that fertilization rates are not affected in patients with laparoscopic cystectomy. Findings revealed that parameters for ovarian stimulation and fertility outcome are similar in both groups. However, women with surgically treated endometrioma had a lower oocyte retrieval and required higher $\mathrm{FSH}$ stimulation compared with unexplained infertility, but results are without any statistical significance. Therefore, we conclude that the operative treatment of ovarian unilateral endometrioma has no adverse effect on IVF outcome.

\section{Limitation}

By limiting the study to a fresh ET cycle, it was not possible to assess the cumulative outcome including the use of all frozen embryos. The findings of the study should be confirmed in future large trials with the possibility to include additional significant covariates in the statistical model.

\section{Conclusion}

We can conclude that surgery does not adversely affect the examined parameters for ovarian stimulation and the outcome of IVF. The theoretical risk of loss of ovarian tissue during surgery remains but can be avoided by carefully preserving normal ovarian tissue. Surgical management should be individualized for women with endometriomas and attention to be given to the preoperative ovarian reserve status. Comparing the outcome of IVF/ET results, there was no significant difference in women with laparoscopic treated endometrioma and nonendometriosis group.

The most important clinical outcome is the live birth rate, which was similar in both groups. Finally, the efficiency of laparoscopic procedures in managing endometriosis-related infertility is crucial by return live birth rate in the level expected in the women without endometriosis.

It is important to note that most studies agree with the fact that individual patient factors have the most important role in the IVF outcome. According to that, individual treatment based on the expertise of the physician and needs of the patient is recommended in patients with endometriosis-associated infertility. 


\section{Acknowledgment}

The authors would like to thank Prof. Nikola Orovchanec, PhD, MD, kindly performed statistical analysis.

\section{Authors' Roles}

S.S., G.D., J.S., and M.HL. participated in study design, execution, analysis, manuscript drafting, and critical discussion.

\section{References}

1. Dunselman GA, Vermeulen N, Becker C, Calhaz-Jorge C, D'Hooghe T, De Bie B, et al. ESHRE guideline: Management of women with endometriosis. Hum Reprod. 2014;29(3):400-12. https://doi.org/10.1093/humrep/det457

PMid:24435778

2. Garcia-Velasco JA, Somigliana E. Management of endometriomas in women requiring IVF: To touch or not to touch. Hum Reprod. 2009;24(3):496-501. https://doi.org/10.1093/ humrep/den398

PMid:19056774

3. Coccia ME, Rizzello F, Mariani G, Bulletti C, Palagiano A, Scarselli G. Impact of endometriosis on in vitro fertilization and embryo transfer cycles in young women: A stage-dependent interference. Acta Obstet Gynecol Scand. 2011;90(11):1232-8. https://doi.org/10.1111/j.1600-0412.2011.01247.x

PMid:21793811

4. Garcia-Velasco JA, Fassbender A, Ruiz-Alonso M, Blesa D, D'Hooghe T, Simon C. Is endometrial receptivity transcriptomics affected in women with endometriosis? A pilot study. Reprod Biomed Online. 2015;31(5):647-54. https://doi.org/10.1016/j. rbmo.2015.07.014

PMid:26385059

5. Matalliotakis IM, Cakmak $\mathrm{H}$, Mahutte $\mathrm{N}$, Fragouli $\mathrm{Y}$, Arici $\mathrm{A}$, Sakkas D. Women with advanced-stage endometriosis and previous surgery respond less well to gonadotropin stimulation, but have similar IVF implantation and delivery rates compared with women with tubal factor infertility. Fertil Steril. 2007;88(6):1568-72. https://doi.org/10.1016/j.fertnstert.2007.01.037

PMid:17349642

6. Jacobson TZ, Barlow DH, Koninckx PR, Olive D, Farquhar C. Laparoscopic surgery for subfertility associated with endometriosis. Cochrane Database Syst Rev. 2002;1:CD001398. https://doi.org/10.1002/14651858.cd001398

PMid:20091519

7. Benschop L, Farquhar C, van der Poel N, Heineman MJ. Interventions for women with endometrioma prior to assisted reproductive technology. Cochrane Database Syst Rev. 2010;11:CD008571. https://doi.org/10.1002/14651858.cd008571 PMid:21069706

8. Kennedy S, Bergqvist A, Chapron C, D'Hooghe T, Dunselman G, Greb R, et al. ESHRE guideline for the diagnosis and treatment of endometriosis. Hum Reprod. 2005;20(10):2698-704. https:// doi.org/10.1093/humrep/dei135

PMid:15980014

9. Vassilopoulou L, Matalliotakis M, Zervou MI, Matalliotaki C, Spandidos DA, Matalliotakis I, et al. Endometriosis and in vitro fertilization. Exp Ther Med. 2018;16(2):1043-51. https://doi. org/10.3892/etm.2018.6307

PMid:30116357

10. Practice Committee of the American Society for Reproductive Medicine. Endometriosis and infertility: A committee opinion. Fertil Steril. 2012;98(3):591-599.

PMid:22704630

11. Harb H, Gallos I, Chu J, Harb M, Coomarasamy A. The effect of endometriosis on in vitro fertilization outcome: A systematic review and meta-analysis. BJOG. 2013;120(11):1308-1320. https://doi.org/10.1111/1471-0528.12366 PMid:23834505

12. Jones CJ, Inuwa IM, Nardo LG, Litta P, Fazleabas AT Eutopic endometriom from women with endometriosis shows altered ultrastucture and glycosylation compared to that from healthy controls-a pilot observation study. Reprod Sci. 2009;16(6):559-72. https://doi.org/10.1177/1933719109332825 PMid: 19282503

13. Miller DL, Jones CJ, Aplin JD, Nardo LG. Altered glycosylation in peri-implantation phase endometrium in women with stage II and IV endometriosis. Hum Reprod. 2010:25(2):406-11. https:// doi.org/10.1093/humrep/dep401

PMid:19910324

14. Bulun SE, Cheng YH, Yin P, Imir G, Utsunomiya H, Attar E, et al. Progesteron resistance in endometriosis: Link to failure to metabolize estradiol. Mol Cell Endocrinol. 2006;248(1-2):94-103. https://doi.org/10.1016/j.mce.2005.11.041 PMid:16406281

15. Hamdan M, Dunselman G, Li TC, Cheong Y. The impact of endometrioma on IVF/ICSI outcomes: A systematic review and meta-analysis. Hum Reprod Update. 2015;21(6):809-25. https:// doi.org/10.1093/humupd/dmv035 PMid:26168799

16. Muteshi CM, Ohuma EO, Child T, Becker CM. The effect of endometriosis on live birth rate and other reproductive outcomes in ART cycles: A cohort study. Hum Reprod Open. 2018;4:1-7. https://doi.org/10.1093/hropen/hoy016

17. Cranney R, Condous G, Reid S. An update on the diagnosis, surgical management, and fertility outcomes for women with endometrioma. Acta Obstet Gynecol Scand. 2017;96(6):633-643. https://doi.org/10.1111/aogs.13114 PMid:28186620

18. Dechanet C, Rihaoui S, Reyftmann L, Hedon B, Hamamah S, Dechaud $\mathrm{H}$. Endometriosis and fertility: Results after surgery and Assisted Reproductive Technology (ART). Gynécol Obstét Fertil. 2011;39(1):3-7. https://doi.org/10.1016/j.gyobfe.2010.08.018 PMid:21183384

19. Kolanska K, Cohen J, Bendifallah S, Selleret L, Antoine JM, Chabbert-Buffet, et al. Pregnancy outcomes after controlled ovarian hyperstimulation in women with endometriosisassociated infertility: $\mathrm{GnRH}$-agonist versus $\mathrm{GnRH}$-antagonist. J Gynecol Obstet Hum Reprod. 2017;46:681-6. https://doi. org/10.1016/j.jogoh.2017.09.007

20. Park HJ, Kim H, Lee GH, Yoon TK, Lee WS. Could surgical management improve the IVF outcomes in infertile women with endometrioma? A review. Obstet Gynecol Sci. 2019;62(1):1-10. PMid:30671388

21. Coccia ME, Rizzello F, Cammilli F, Bracco GL, Scarselli G. Endometriosis and infertility Surgery and ART: An integrated approach for successful management. Eur J Obstet Gynecol 
Reprod Biol. 2008;138(1):54-9. https://doi.org/10.1016/j. ejogrb.2007.11.010

PMid:18243485

22. Crochet $P$, Lathi $R$, Dahan $M$, Ocampo J, Nutis $M$, Nezhat $C$. Control-matched surgical evaluation of endometriosis progression after IVF: A retrospective cohort study. Minerva Ginecol. 2016;68(5):481-6. https://doi.org/10.1016/j.fertnstert.2007.07.988 PMid:26824508

23. Guo $H$, Wang $Y$, Chen $Q$, Chai $W$, Sun $L$, Ai A, et al. Use of medroxyprogesterone acetate in women with ovarian endometriosis undergoing controlled ovarian hyper-stimulation for in vitro fertilization. Sci Rep. 2017;7:11927. https://doi. org/10.1038/s41598-017-12151-7

PMid:28931865

24. Alkudmani AB, Gat I, Buell D, Salman J. In vitro fertiliza-tion (IVF) success rates after surgically treated endometriosis and effect of time interval between surgery and IVF. J Minim Invasive Gynecol. 2018;25(1):99-104. https://doi.org/10.1016/j. jmig.2017.08.641

\section{PMid:28807807}

25. Leonardi M, Papaleo E, Reschini M, Pagliardini L, Benaglia L, Candotti G, et al. Risk of miscarriage in women with endometriosis: Insights from in vitro fertilization cycles. Fertil Steril. 2016;106(2):386-92. https://doi.org/10.1016/j. fertnstert.2016.03.047

PMid:27090864

26. Boujenah J, Santulli P, Argent EM, Decanter C, Chauffour C Poncelet P. First line management without IVF of infertility related to endometriosis: Result of medical therapy? Results of ovarian superovulation? Results of intrauterine insemination? CNGOFHAS Endometriosis Guidelines. Gynécol Obstét Fertil Sénol. 2018;46:331-7. https://doi.org/10.1016/j.gofs.2018.02.010 PMid:29551300

27. Li X, Zeng C, Zhou YF, Yang HX, Shang J, Zhu SN, et al. Endometriosis fertility index for predicting pregnancy after endometriosis surgery. Chin Med J (Engl). 2017;130(16):1932-7. https://doi.org/10.4103/0366-6999.211892

PMid:28776545 